\title{
OBSERVACIONES ADICIONALES SOBRE LA HISTORIA NATURAL DE LA RANA GLADIADORA HYPSIBOAS PUGNAX (O. SCHMIDT, 1857), EN EL CARIBE COLOMBIANO
}

\author{
ADDITIONAL OBSERVATIONS ON NATURAL HISTORY OF GLADIATOR FROG, \\ HYPSIBOAS PUGNAX (O.SCHMIDT, 1857), IN THE COLOMBIAN CARIBBEAN
}

\author{
ADDENDA ET CORRIGENDA
}

APORTES PARA LA IDENTIFICACIÓN DE LAS RANAS GLADIADORAS DEL GÉNERO HYPSIBOAS (WAGLER, 1830); (ANURA: HYLIDAE), PRESENTES EN LAS TIERRAS BAJAS DEL CARIBE COLOMBIANO

CONTRIBUTIONS FOR THE IDENTIFICATION OF GLADIATOR FROGS FROM GENUS HYPSIBOAS (WAGNER, 1830); (ANURA: HYLIDAE), PRESENT IN THE COLOMBIAN CARIBBEAN LOWLANDS

MENDOZA ROLDAN, JUAN SALVADOR, Biol.

Universidad de los Andes, Colección de herpetología, Colombia.

\section{Palabras Clave:}

Hypsiboas, Depredadores, Crustaceae, Addenda et corrigenda, Superficie antero ventral de la tibia, Pigmentación.

\section{Resumen}

En esta nota se documenta dos eventos de la historia natural de Hypsiboas pugnax en el Caribe colombiano, se describe una nidada en el municipio de Rio Ancho, Guajira, hallado durante el mes de marzo de 2015, y adicionalmente un evento de depredación, que involucra a un macho adulto de esta rana, la presa, y un camarón nativo. En esa nota se incluye una importante corrección a tener en cuenta sobre la publicación: "Aportes para la identificación de las ranas gladiadoras del género Hypsiboas (Wagler, 1830); (anura: Hylidae), presentes en las tierras bajas del Caribe colombiano", publicada en RECIA en 2014. Se aclara la posición topológica del carácter denominado "pigmentación sobre la superficie ventral anterior de la tibia" carácter que fue ilustrado erróneamente en la publicación referida, por lo que se presenta la respectiva Addenda et corrigenda.
Key words:

Nesting,

Predators,

crustaceans,

Addenda et corriegenda,

Antero ventral surface of the Tibia, Pigmentation.

INFORMACIÓN

Recibido: 15-03-2015;

Aceptado: 22-06-2015.

Correspondencia autor:

is.mendoza122@uniandes.edu.co,

viperiuan@gmail.com

\section{Abstract}

The present note documents two natural history events of Hypsiboas pugnax. A nesting event was observed and described in Rio Ancho, Guajira Colombiana, during the month of March, 2015. Additionally a predation event was observed; it involved a carnivorous native prawn and an adult $H$. pugnax, its prey. This note includes an important correction on the previously published: "Contributions for the identification of gladiator frogs from genus Hypsiboas (Wagner, 1830); (Anura: Hylidae), present in the Colombian Caribbean lowlands", published in RECIA on 2014. This note is intended to clarify the use of the character named as: "Presence of pigmentation over the anterior region of the tibia", this character was imprecisely illustrated in the referred publication, reason why this Addenda et corrigenda is needed. 


\section{Nidada en Hypsiboas pugnax}

Durante trabajo de campo realizado en el mes de Marzo de 2015, en el Corregimiento de Rio Ancho, Municipio de Dibulla, Guajira Colombiana; se realizó la observación de una nidada de Hypsiboas pugnax (Figs. 1 y 2). La postura constaba de 980 huevos, cada huevo con un diámetro aproximado de $1,3 \mathrm{~mm}$. Esa postura se encontraba dentro de un nido que consistía de una pequeña depresión al borde de la quebrada con una excavación de $15 \mathrm{~cm}$ de largo y $14.91 \mathrm{~cm}$ de ancho y dos centímetros de profundidad. El macho constructor de este nido se ha encargado de hacer con las patas traseras, una rampa o muro de contención en la cara del nido que da hacia la quebrada (Fig. 1).

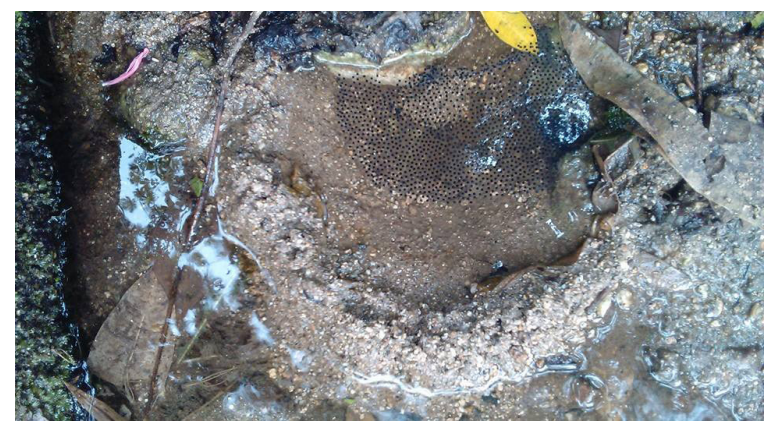

Figura 1. Nido de Hypsiboas pugnax donde se observa la presencia de una pared o rampa protectora en la cara que da hacia la quebrada.

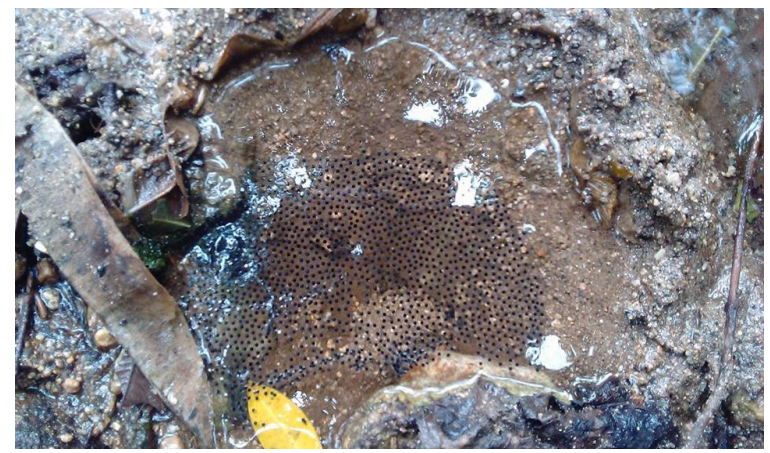

Figura 2. Detalle de la postura de Hypsiboas pugnax; Guajira, Municipio de Dibuya, Corregimiento de Rio Ancho.

Observaciones realizadas por GALVIS-PEÑUELA et al. (2011); para las poblaciones de $H$. pugnax en los montes de Oca, Guajira, Colombiana; incluyen algunos aspectos sobre el comportamiento de nidificación. Comentan, que los machos de esta especie construyen nidos en el barro a las orilla de las quebradas, los cuales defienden. Adicionalmente describen el ritual de apareamiento, que involucra el comportamiento de lucha que exhiben las ranas gladiadoras. En este caso las hembras se aproximan a los nidos atraídas por el canto del macho, luego se da una lucha entre ambos, donde la hembra intentará empujar al macho hasta sacarlo fuera de su nido.
En esta especie la selección por parte de la hembra se basa además del canto, en la fuerza que tenga el macho para mantenerse posicionado dentro del nido, que será una medida directa de su aptitud Darwiniana (KLUGE, 1981). CHACÓN-ORTIZ et al. (2004) describen que los nidos en $H$. pugnax son de forma circular a elíptica; con un volumen de $1.476,89 \mathrm{~cm}^{3}$ y un número de 1.896 huevos. Ellos comentan que la especie los construye de manera facultativa, dependiendo de las condiciones del micro hábitat, su construcción se da en ambientes de quebrada, con sustratos arenosos o arcillosos. El cuidado parental es realizado enteramente por el macho, que vigila el nido durante la noche. La reproducción de esta especie en Venezuela se concentra en la época de sequía (febrero), donde se ha observado que el desarrollo larval, dura 95 días a una temperatura de $21^{\circ} \mathrm{C}$. La formación de las branquias se origina a los siete días, seguida por la aparición del espiráculo a los 22 días; la eruptopodia se da a los 45 días. Por último la reabsorción de la cola se da a los 92 días de desarrollo (CHACON-ORTIZ et al. 2004).

El diseño del nido observado para $H$. pugnax, muestra una rampa o muro en la cara que da hacia la quebrada (Fig. 1). Comparativamente, este diseño muestra diferencias con lo reportado anteriormente para el del nido de $H$. Rosenbergi, donde se observaron nidos elaborados en charcas dentro del bosque que presentaban diseños alargados y profundos junto con la ausencia de una rampa en su perímetro (ver Figs. 4B y $5 A$ en MENDOZA, 2014). BREDER (1927) concluye que $H$. rosenbergi, construye los nidos con rampas y barreras en ambientes de quebrada solo cuando se encuentra ante la presencia de peces predadores de huevos y larvas (KLUGE, 1981).

En otras localidades como Puerto triunfo Antioquia se observaron amplexos de $H$. Pugnax durante el mes de diciembre de 2012, estos amplexos se realizaban dentro del agua y sobre la vegetación rasante en la orilla de cuerpos lenticos sin evidencia de la presencia de nidos. La variación intra-especifica en la presencia o ausencia del nido y su diseño entre las especies genero Hypsiboas, sugiere la existencia de un alto grado de plasticidad adaptativa en este comportamiento. Las ranas de la familia Hylidae explotan reproductivamente tanto quebradas (permanentes y temporales), como charcas. Ambos son ambientes acuáticos muy distintos en su composición de especies depredadoras, tanto vertebradas como invertebradas (WILSON et al. 2015). En el género Hypsiboas, la construcción del nido se encuentra relacionada con la inversión del cuidado parental, comportamiento que ha evolucionado en base a la presión ejercida por la depredación sobre etapas de vida vulnerables como larvas y huevos.

\section{Depredación en $\boldsymbol{H}$. pugnax}

Durante trabajo de campo realizado en enero de 2014 en la vereda el Corral de San Luis, Municipio de Tubará, departamento del Atlántico, se realizaron observaciones y fotografías sobre un evento de depredación que 
involucra a un individuo macho adulto de $H$. pugnax y un individuo de camarón nativo Macrobrachium carcinus (LINÉE, 1758). Este camarón capturo a la rana en la orilla de una pequeña quebrada, la mantuvo sumergida hasta darle muerte, y poder alimentarse de ella. La depredación inducida por crustáceos decápodos como la especie invasora Procambarus clarkii (GIRARD, 1852), se ha descrito para las larvas y huevos de Bufo calamita en España, donde junto con otras especies de crustáceos, causan hasta el $95 \%$ de la depredación de los huevos (PORTHEAULT et al., 2007). Las observaciones de depredación por parte de crustáceos decápodos sobre anuros adultos son poco frecuentes y raramente documentadas, algunas de ellas incluyen al cangrejo de agua salada Leptograpsus variegatus (FABRICIUS, 1793), para el cual se reporta el uso alimenticio de post-metamorfos de la rana Litoria aurea (PYKE et al., 2013), algunas especies de crustáceos se alimentan de ranas de la familia Dendrobatidae, para lo cual remueven primero la piel venenosa de la rana (AMEZQUITA, A. Obs. Pers.). Las ranas del género Hypsiboas son presa común de un gran espectro de depredadores vertebrados como aves, reptiles y primates (ROCHA et al., 2014; GALVIS-PEÑUELA et al., 2011; KLUGE, 1981). Artrópodos como arácnidos, crustáceos e insectos de los Ordenes Odonata y Hemíptera; depredan frecuentemente los huevos y larvas de estas ranas, pese a esto, se considera que la depredación sobre adultos resulta como un uso oportunista de este recurso alimenticio. (WILSON et al., 2015).

Addenda et corrigenda: En el artículo mencionado en esta nota, publicado en RECIA en 2014 , se hace referencia a un carácter nombrado como el grado de extensión de la pigmentación en la región tibial anterior. Este carácter fue nombrado varias veces refiriéndose a una topología morfológica errada, debido a que la fotografía muestra a un animal en posición dorsal (Fig. 2 y Fig. 3, Fig. 6 C en MENDOZA, 2014). En estas figuras, se observa el patrón de pigmentación, solo sobre la superficie dorsal del fémur y de la tibia. En la Fig. 8B, se muestra la extremidad posterior de $H$. pugnax y no propiamente de la tibia en su totalidad como menciona la leyenda, de esta, solo se observa el extremo proximal de la superficie ventral. En esta nota de corrección destacamos que uno de los caracteres mas informativo en la morfología externa y por ende en la identificación

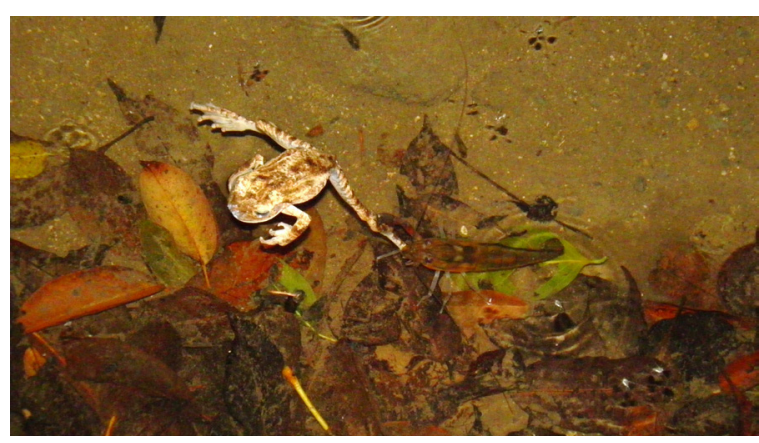

Figura 3. Camarón nativo Macrobrachium carcinus depredando a un individuo de Hypsiboas pugnax en la quebrada de la reserva de la sociedad civil La Esmeralda, Vereda de Corrales de San Luis, Municipio de Tubará, Atlántico.

en campo es el descrito anteriormente por CUENTAS et al. (2002), en esta publicación se esquematiza las características del patrón de barreteado en una ilustración que compara la extensión de la pigmentación en la superficie ventral del fémur en $H$. pugnax, $H$. crepitans y $H$. rosenbergi El patrón de barreteado y la pigmentación es una coloración predominantemente dorsal en las extremidades posteriores, donde suele extenderse hacia la región femoral ventral. En $H$. Pugnax está pigmentación cubre parte de la superficie ventral del fémur y se extiende hasta la región antero ventral de la tibia (DUELLMAN, 2001).

Agradecimientos: Eternamente agradecido con mi familia del Corral de San Luis Beltrán, Tubará, Atlántico, que han apoyado la investigación en su zona de reserva forestal comunitaria el Chorro- la Esmeralda, desde 2004. Gracias a eventos como el IV Congreso Colombiano de Zoología, encontré críticas y comentarios constructivos a este trabajo, por lo que también quiero agradecer a los colegas y amigos que se preocuparon por intentar usar la clave en campo y observar la necesidad de aclarar el uso de este carácter. Especialmente gracias a Beto Rueda por su invitación a Rio Ancho, pese a todo su apoyo siempre ha sido incondicional.

\section{Referencias}

CHACÓN-ORTIZ, A.; DÍAZ DE PASCUAL, A.; GODOY, F. 2004. Aspectos reproductivos y desarrollo larval de Hyla pugnax (Anura:Hylidae) en el Piedemonte Andino de Venezuela. Revista de la. Academia Colombiana de Ciencias 28(108): 391-402.

DUELLMAN, W.E. 2001. Hylid frogs of Middle America. Ithaca, NY: Society for the Study of Amphibians and Reptiles. NY. USA.

GALVIS-PEÑUELA, P.A.; MEJIA-TOBON, A.; RUEDA-ALMONACID, J.V. 2011. Fauna Silvestre de la Reserva Forestal Protectora Montes de Oca, La Guajira, Colombia. Corpoguajira. Colombia. 
KLUGE, A.G. 1981. The Life History, Social Organization, and Parental Behavior of Hyla rosenbergi Boulenger, a Nest-Building Gladiator Frog. Miscellaneous Publications. Museum of Zoology, University of Michigan. No. 160. Michigan, USA.

PORTHEAULT, A.; DÍAZ-PANIAGUA, C.; GÓMEZ-RODRÍGUEZ, C. 2007. Predation on amphibian eggs and larvae in temporary ponds: The case of Bufo calamita in Southwestern Spain. Revue D Ecologie-la Terre Et La Vie. 62: $315-322$.

PYKE, G.H.; AHYONG, S.T.; FUESSEL, A.; CALLAGHAN, S. 2013. Marine crabs eating freshwater frogs: Why are such observations so rare? Herpetology Notes 6:195-199.

ROCHA, R.; Lopez-Baucells, A. 2014. Predation attempt of Hypsiboas boans (Anura: Hylidae) by Helicops angulatus (Squamata: Dipsadidae) withnotes on defensive behavior. ALYTES 30:78-81.

VALENCIA, D.; CAMPOS, M.R. 2007.Freshwater prawns of the genus Macrobrachium Bate, 1868 (Crustacea: Decapoda: Palaemonidae) of Colombia. Zootaxa 1456: 1-44.

WILSON, N. J.; SEYMOUR, J.E.; WILLIAMS, C.R. 2015. Predation of two common native frog species (Litoria ewingi and Crinia signifera) by freshwater invertebrates. Australian Journal of Zoology 62(6):483-490. 